\title{
Review: \\ Potentiality of Graphene Oxide and Polyoxometalate as Radionuclides Adsorbent to Restore the Environment after Fukushima Disaster: A Mini Review
}

\author{
Bangun Satrio Nugroho ${ }^{1,2}$, Muh Nur Khoiru Wihadi ${ }^{3}$, Fabian Grote ${ }^{4}$, \\ Siegfried Eigler ${ }^{4}$, and Satoru Nakashima ${ }^{1,2,5^{*}}$ \\ ${ }^{1}$ Radioactivity Environmental Protection Course, Phoenix Leader Education Program, Hiroshima University, \\ 1-1-1 Kagamiyama, Higashi-Hiroshima 739-8524, Japan \\ ${ }^{2}$ Department of Chemistry, Graduate School of Science, Hiroshima University, \\ 1-3-1 Kagamiyama, Higashi-Hiroshima 739-8526, Japan \\ ${ }^{3}$ Department of Chemistry, Faculty of Military Mathematics and Natural Science, Indonesia Defense University, \\ Kawasan Indonesia Peace and Security Center (IPSC), Bogor 16810, Indonesia \\ ${ }^{4}$ Institut fur Chemie und Biochemie, Freie Universitat Berlin, Takustrabe 3, 14195 Berlin, Germany \\ ${ }^{5}$ Natural Science Center for Basic Research and Development, Hiroshima University, \\ 1-4-2 Kagamiyama, Higashi-Hiroshima 739-8526, Japan
}

${ }^{*}$ Corresponding author:

email:snaka@hiroshima-u.ac.jp

Received: October 9, 2020

Accepted: December 4, 2020

DOI: $10.22146 /$ ijc.60493

\begin{abstract}
This paper discusses the promising candidate of excellent materials, graphene oxide (GO) and polyoxometalates (POMs), for radionuclide adsorbent. In this perspective, the unique properties of GO and POMs make them ideal candidates for developing new composites having the ability to adsorb radionuclides, and several essential things are reviewed. First, the anchoring mechanism to deposit POM on the GO surface area by (i) carboxylation method, (ii) covalent bonding, and (iii) impregnation method. Second, the radionuclides removal mechanism is described in several systems: (i) coagulation, (ii) electrostatic interaction, (iii) ion trapping, and (iv) $\mathrm{H}^{+}$-exchange. Third, the experimental condition that employed to enlarge the sorption capacity such as (i) $p H$ adjustment, (ii) employing multiple oxidations, and (iii) cation charge. A thorough understanding of the POM-anchored GO material can pave the way for future research on similar materials. It can also help in understanding the nature of the interactive collaboration present between GO and POM.
\end{abstract}

Keywords: adsorbent; Fukushima disaster; graphene oxide; polyoxometalates; radionuclides

\section{- INTRODUCTION}

The Fukushima Daiichi nuclear power plant (FDNPP) disaster (March 2011) affected the biotic and abiotic environment. FDNPP accident released radioactive elements that possess long half-lives (30 years for cesium $\left.\left({ }^{137} \mathrm{Cs}\right)\right), \beta$-decay to barium $\left({ }^{137} \mathrm{Ba}\right)$, and emit $\gamma$ radiation. It has been reported that the soil present around FDNPP and the soil in the neighboring prefectures were contaminated with approximately $100,000 \mathrm{MBq} / \mathrm{km}^{2}$ and
$10,000 \mathrm{MBq} / \mathrm{km}^{2}$ of ${ }^{137} \mathrm{Cs}$, respectively [1]. The soil surface area was the most contaminated [2]. It has been predicted that several radionuclides, such as cesium $\left({ }^{137} \mathrm{Cs},{ }^{136} \mathrm{Cs}\right.$, $\left.{ }^{134} \mathrm{Cs}\right)$, strontium $\left({ }^{90} \mathrm{Sr},{ }^{89} \mathrm{Sr}\right)$, uranium $\left(\mathrm{UO}^{2+}\right)$, iodine $\left({ }^{131} \mathrm{I}\right)$, thorium $\left({ }^{90} \mathrm{Th}\right)$, barium $\left({ }^{140} \mathrm{Ba}\right)$, lanthanum $\left({ }^{140} \mathrm{La}\right)$, and tritium $\left({ }^{3} \mathrm{H}\right)$ are present. An analysis by Tokyo Electric Power Company (TEPCO), an electric power company, revealed that the major part of the fuel (in unit 1 nuclear reactor) was present in its molten state [3]. The radioactive isotopes released 
during the Fukushima accident and predominantly responsible for the observed radioactivity were the radioactive isotopes of Sr and Cs. It has been reported that $\mathrm{Sr}$ exhibits a faster rate of migration compared to other elements [4]. It has been observed that Cs $\left({ }^{137} \mathrm{Cs},{ }^{136} \mathrm{Cs}\right.$, ${ }^{134} \mathrm{Cs}$ ) and ${ }^{131} \mathrm{I}$ become the more radioactive elements that were released from the fuel if compared with $\mathrm{Sr}\left({ }^{90} \mathrm{Sr},{ }^{89} \mathrm{Sr}\right)$ [5-6].

It is necessary to estimate the quantity of radionuclides and chemicals released during the accident to determine the immediate and long-term effects of radioactivity on the environment and health of organisms. Researchers have studied the mobility of radioactivity, especially the uptake of radioactive nuclides by plants [7]. The uptake of radioactive nuclides by plants can potentially lead to food contamination that can eventually lead to genome damage or cancer in human beings. Further investigation also reported that slightly high levels of radioactive cesium were detected in some rice varieties grown in areas approximately $60 \mathrm{~km}$ northwest of the nuclear reactor plant, a few years after the accident in Fukushima City [8]. Researchers have reported that the transfer factor (from the soil to the rice) of radioactive cesium depended on the oxidizing or reducing soil atmosphere [9]. The penetration depth (in soil) of radiocesium is influenced by soil characteristics (such as the particle size, organic carbon content, and clay contents) [10]. One of the key factors influencing the penetration depth of radiocesium in the soil is the amount of medium-sized sand particles present in the soil [11]. Moreover, an investigation was carried out with soil samples collected from the area around the Hibara Lake (Yama gun, Fukushima prefecture), situated $100 \mathrm{~km}$ away from FDNPP, revealing that radionuclides (originating from the Fukushima prefecture) were present in the sediments deposited in the shallow areas of the lake. ${ }^{134} \mathrm{Cs}$ and ${ }^{137} \mathrm{Cs}$ isotopes were detected $(4.5$ and $5.2 \mathrm{~Bq} / \mathrm{kg}$, respectively) in the soil of these areas. The results revealed the long-distance migration of radioactive cesium. The high solubility of radiocesium in water assists soil penetration. Compared to the process of direct deposition of the nuclides (originating from the exhaust of the accident), the process of nuclide transport by rivers into lakes (and its subsequent sedimentation) is more likely to occur [12]. Shizuma et al. investigated the quality of drinking water in the Haramachi district (Minamisoma City). The authors detected dissolved ${ }^{137} \mathrm{Cs}$ in tap water and groundwater. The maximum concentration of ${ }^{137} \mathrm{Cs}$ was approximately $300 \mathrm{mBq} / \mathrm{L}$ in August 2013 and 27 $\mathrm{mBq} / \mathrm{L}$ in September 2014 [13]. The degree of contamination was not solely dependent on the distance of the place under observation from the area of the nuclear accident. Other factors, such as weather conditions (such as wind direction and precipitation), also affected the degree of soil contamination [14].

These results revealed that radionuclide contamination, which can potentially impact human lives, is a severe environmental problem. The contamination had spread far and wide, away from the Fukushima nuclear power plant. Therefore, it is necessary to decontaminate the environment effectively. A few decontamination strategies, such as removing the litter layer and superficial soil layer in the forests, have already been implemented [15-16]. Decontamination has also been attempted by scraping off the topsoil and cleaning tree barks. The reverse plowing technique has also been used for the same aim [17]. Recently, researchers have attempted to synthesize high-quality adsorbent nanomaterials that can potentially remove radionuclides from the environment. Graphene oxide (GO) and polyoxometalate (POM) derivatives are the two classes of materials that have recently attracted immense attention. GO has been the focus of research as it has its application in various fields, such as biomedicine [18-19] and environmental protection [20$22]$. It has also been used to develop energy storage devices [23], polymer composites [24], and gas sensors [25]. The properties of GO can be tuned by systematically functionalizing the material. This can lead to improved performance of the materials [26]. Similar to GO, the POM derivatives are high-performance adsorbent nanomaterials. The synthesis of materials that can function as radionuclide adsorbents, such as $\mathrm{H}$-titanate nanotubes [27] and phlogopite [28], has been reported. However, not all the reported materials are excellent adsorbents. Therefore, a potential material that 
can function as a highly efficient radionuclide adsorbent should be identified. This paper aims to highlight the potential of GO and POMs as radionuclide adsorbents to restore environmental conditions.

\section{- GRAPHENE OXIDE (GO)}

GO is a promising candidate that can be potentially used for adsorbing radionuclides. The epoxide and hydroxyl groups are the two major functional groups present on both sides of the basal plane of the GO sheets [26]. GO contains numerous structural defects. Other functional groups, such as carbonyl groups, phenol-like groups, and carboxyl groups, are present at the rims of these defects. The particle size of GO plays an important role in determining the functional group composition of the material [29-30]. Therefore, the adsorption capacity of GO is dictated by the accessible surface area. Thus, it is important to understand the structural characteristics of GO to gain insight into the adsorption process.

Recently, researchers have followed the combined method proposed by Grote et al. and attempted the fabrication of GO. The oxo-functionalized graphene bears an almost intact hexagonal carbon lattice (unlike the conventional GO material fabricated following Hummer's method) [31]. The fabrication method involves a three-step process (the cold, medium, and hot stages). The transition from one stage to the other is accompanied by a change in color of the reaction mixture, which indicates the successful completion of each stage (Fig. 1(a), (b), and (c)) [32]. The fabrication of a single (or a few) layers of GO can be achieved through the processes of chemical oxidation and exfoliation [33]. The transmission electron microscopy (TEM) and scanning electron microscopy (SEM) techniques were used to visualize the surface morphology of GO (Fig. 1(d) and (e)). The SEM and TEM images revealed the presence of wrinkled and overlapping GO sheets. These properties were a result of the hydrogenbonding interactions present between the surface functional groups. The wrinkled and overlapped samples exhibit different optical and electrical properties [34]. Guerrero-Contreras and Caballero-Briones reported that

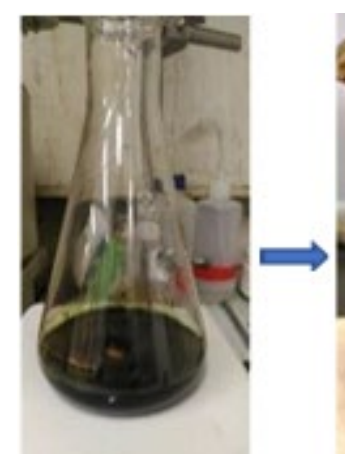

(a) cold

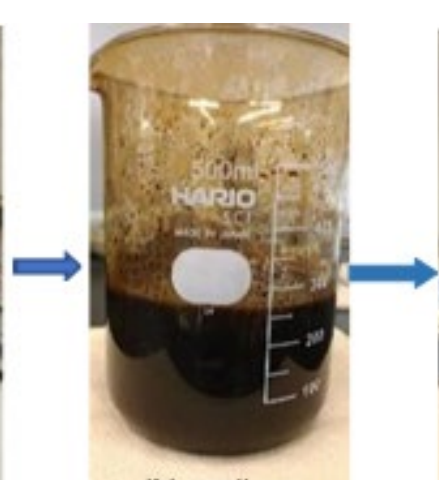

(b) medium

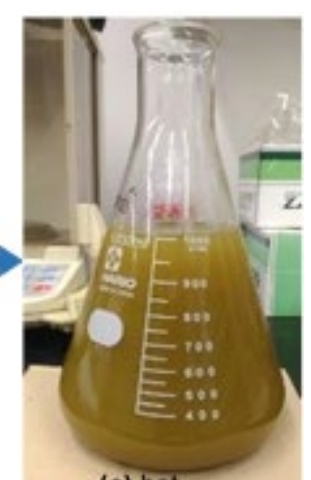

(c) hot

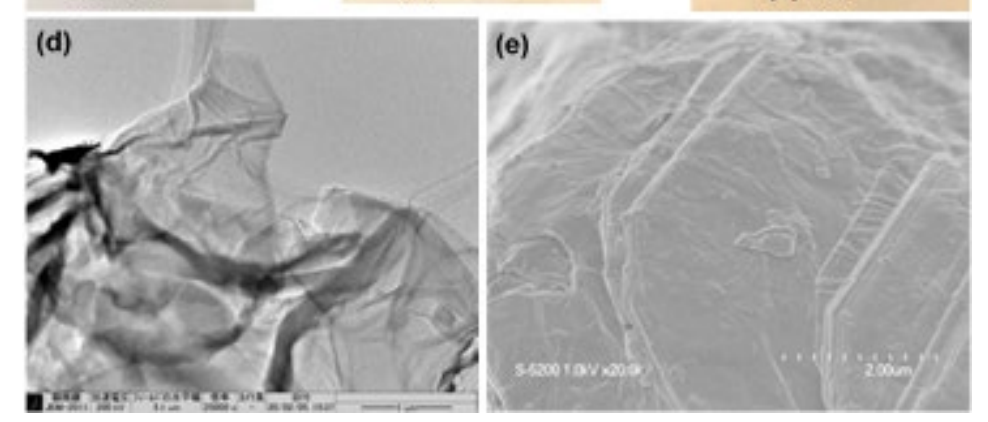

Fig 1. Synthesis of GO using the modified Hummer's method. (a) Cold stage (Blackish green), (b) Medium stage (Brown), (c) Hot stage (Golden yellow particles of GO), (d) Transmission electron microscopy (TEM) image of GO, and (e) Scanning electron microscopy (SEM) image of GO. Image is taken from personal data based on an experimental study 
the degree of GO oxidation dictates this particular characteristic, especially the surface chemistry of the sheets. These properties can be exploited by decorating GO [35].

The characteristic peaks of different functional groups were observed in the Fourier transform infrared (FTIR) spectral profiles (Fig. 2). The peaks observed at $1057,1230,1730$, and $3424 \mathrm{~cm}^{-1}$ corresponded to the stretching vibrations of $\mathrm{C}-\mathrm{O}, \mathrm{C}-\mathrm{OH}, \mathrm{C}=\mathrm{O}$, and $-\mathrm{OH}$ groups, respectively. The peak corresponding to the unoxidized graphitic area was observed at $1618 \mathrm{~cm}^{-1}$ [36].

The Fukushima disaster of 2011 led researchers to focus on developing new materials capable of adsorbing radioactive elements. Experiments were conducted to investigate the effectiveness of $\mathrm{GO}$ as radionuclide (especially actinide) adsorbents. The results revealed that the process of coagulation contributed significantly to the adsorption process. Cation-induced coagulation leads to the quick precipitation of GO. The coagulation of GO (in the presence of cations) is affected by $\mathrm{pH}$. Cations with different charges behave differently under the same $\mathrm{pH}$ conditions. For example, sodium $\left(\mathrm{Na}^{+}\right)$, calcium $\left(\mathrm{Ca}^{2+}\right)$, and lanthanide $\left(\mathrm{Eu}^{3+}\right)$, at the same $\mathrm{pH}$, exhibited different critical coagulation concentration (CCC). The changes visible to the naked eye were observed. The interactions present in the GO sheets impart the GO layers with improved adsorption properties (compared to bentonite clays and activated carbon) [37].

As commonly used for cationic radionuclides, bentonite clays and activated carbon have different physical characteristics. Bentonite consists mostly of montmorillonite (a three-layered mineral) having octahedral and tetrahedral sheets [38]. Activated carbon is a non-porous material possessing a large specific surface area. However, functional groups are absent. Therefore, researchers have attempted to functionalize the surface of activated carbon to improve its adsorption ability [39]. Kaewmee et al. reported that the cesium sorption capacity of pristine GO was comparable to the cesium sorption capacity of Prussian blue. It was experimentally demonstrated that the maximum adsorption capacity was approximately $528 \mathrm{mg}$ Cs/g of GO. The sorption ability was tuned by adjusting the $\mathrm{pH}$ of

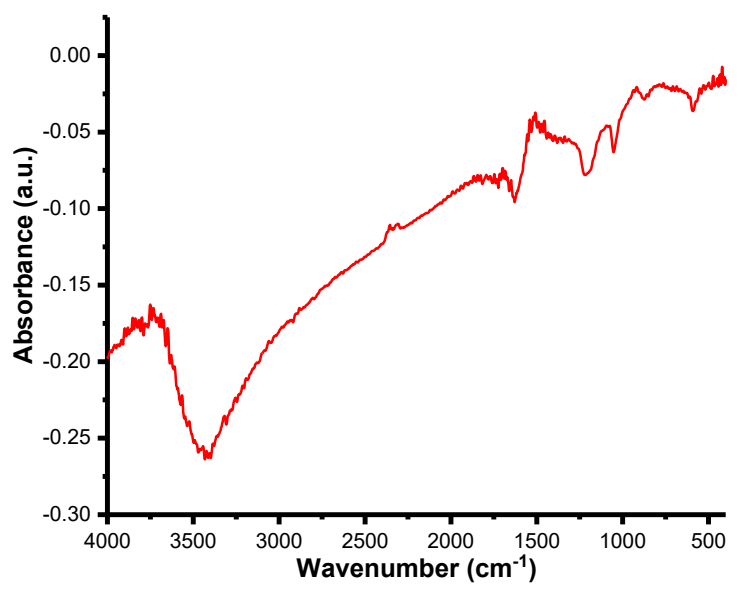

Fig 2. FTIR spectral profile of GO. Graphic took from personal data based on an experimental study

the medium [40]. An interesting phenomenon was observed when the experiments were carried out under a broad range of $\mathrm{pH}$. It experimentally found that the sorption capacity of a magnetic GO nanocomposite, in the $\mathrm{pH}$ range of 5 to 9 , exceeded $90 \%$ (removal of americium $(\mathrm{Am})$ and plutonium $(\mathrm{Pu})$ from solution) [41].

Yang et al. successfully developed a composite consisting of Prussian blue, $\mathrm{Fe}_{3} \mathrm{O}_{4}$ nanoparticles, and $\mathrm{GO}$. It was reported that $\mathrm{GO}$ is an efficient cesium adsorbent. The cesium sorption capacity of the $\mathrm{PB} / \mathrm{Fe}_{3} \mathrm{O}_{4} / \mathrm{GO}$ composite $(55.56 \mathrm{mg} / \mathrm{g})$ was higher than that of the $\mathrm{PB} / \mathrm{Fe}_{3} \mathrm{O}_{4}$ composite $(46.30 \mathrm{mg} / \mathrm{g})$. The successful removal of $\mathrm{Cs}$ (by $\mathrm{H}^{+}$-exchange or ion trapping) can be attributed to the anchoring of Prussian blue and $\mathrm{Fe}_{3} \mathrm{O}_{4}$ nanoparticles on the surface of GO. However, peaks corresponding to $\mathrm{GO}$ were not observed in the PXRD pattern because of the low GO content of the samples [42].

Bubenikova et al. reported that the adsorption capacity of GO could be improved via the carboxylation process (PS_25GO, PS_15GO-COOH, Table 1). The results revealed that the uptake kinetics of strontium(II) was fast. A simple method was used for carboxylation. Multiple carboxylate groups were introduced on the GO surface using a solution of chloroacetic acid in water. Subsequently, a GO-polystyrene (GO-PS) nanocomposite was fabricated to develop the desired material [43].

Recently, researchers have attempted to tune the properties and control the degree of oxidation of the 
Table 1. $\mathrm{Eu}(\mathrm{III}), \mathrm{Sr}(\mathrm{II})$, and $\mathrm{Cs}^{+}$sorption capacity of GO and other materials

\begin{tabular}{lccccc}
\hline \multirow{2}{*}{ Nanocomposite } & \multicolumn{3}{c}{ Maximum sorption capacity $(\mathrm{mg} / \mathrm{g})$} & \multirow{2}{*}{$\mathrm{pH}$ range } & \multirow{2}{*}{ Ref } \\
\cline { 2 - 5 } & $\mathrm{Eu}(\mathrm{III})$ & $\mathrm{Sr}(\mathrm{II})$ & $\mathrm{Cs}$ & & \\
\hline PS_25GO & - & 20.2 & - & $2.0-8.0$ & {$[43]$} \\
PS_15GO-COOH & - & 11.47 & - & $2.0-7.0$ & {$[43]$} \\
GO nanosheet & 175.44 & - & - & 6.0 & {$[21]$} \\
GOnanosheet & 161.29 & - & - & 4.5 & {$[21]$} \\
H-titanates nanotubes short & 22.8 & - & - & 4.2 & {$[27]$} \\
GO & - & - & 528 & 12 & {$[40]$} \\
GO & - & - & 465 & 7 & {$[40]$} \\
Phlogopite & - & - & 14.8 & 2 & {$[28]$} \\
Ca-phlogopite & - & - & 60.4 & 2 & {$[28]$} \\
PANI@GO & - & 266.3 & 121.7 & 3 & {$[22]$} \\
\hline
\end{tabular}

graphene-like materials by tailoring their oxygen content (following specific methods) [44-45]. Novacek et al. reported that a significant increase in the sorption capacity could be achieved by oxidizing GO multiple times. The multiple oxidation process is the key to achieve high oxygen content and significantly increased carboxylic acid content, leading to the significantly high sorption capacity of the material [46]. Researchers have also investigated the role of cation bridging in promoting the formation of GO complexes. The Cs cations were better transport inhibitors at higher concentrations than other monovalent cations. The surface functional groups played an important role in achieving transport inhibition. GO can form inner-sphere complexes with Cs cations [47].

\section{- ANCHORING POLYOXOMETALATE (POM) ON THE SURFACE OF GRAPHENE OXIDE}

POMs are anionic metal oxide clusters consisting of tungsten (W), vanadium (V), molybdenum (Mo), and niobium $(\mathrm{Nb})$ in their high oxidation states. POMs possess interesting properties (such as redox, acidic, photochemical, and magnetic properties) [48]. To date, many families of POMs have been reported [49-50], such as the phosphotungstate family, which is one of the most popular families of POMs. The Keggin-type compound $\left[\mathrm{PW}_{12} \mathrm{O}_{40}\right]^{3-}$ is a member of the phosphotungstate family consisting of one phosphate unit surrounded by 12 tungstate units.

Other members of the family include the Dawsontype compound $\left[\mathrm{P}_{2} \mathrm{~W}_{18} \mathrm{O}_{60}\right]^{6-}$ formed of two phosphate and 18 tungstate units and the Preyssler-type compound $\left[\mathrm{P}_{5} \mathrm{~W}_{30} \mathrm{O}_{110} \mathrm{Na}\left(\mathrm{H}_{2} \mathrm{O}\right)\right]^{14-}$ consisting of five phosphates surrounded by 30 tungstates (Fig. 3). Structurally diverse POMs can find their applications in a wide range of fields. In this mini-review, we have focused on the removal of radionuclides using POM. Recently, it has been investigated if POMs exhibiting the Dawson-type structure can be used to extract thorium and actinium [51]. This work has successfully exploited the coordination

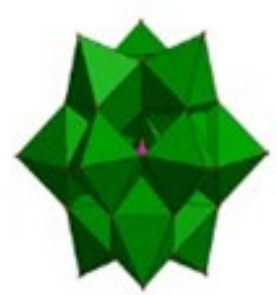

Keggin-type POMs

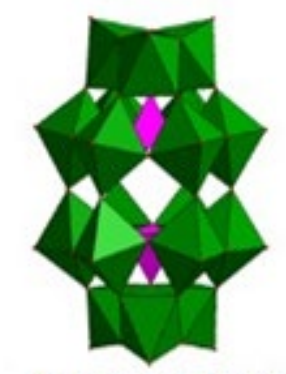

Dawson-type POMS

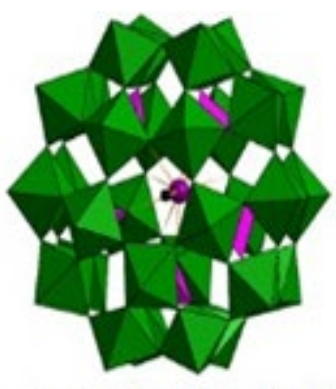

Preyssler-type POMS

Fig 3. Members of the phosphotungstate family of POMs 
chemistry of thorium and actinium and has conducted experiments under acidic conditions (using ion-exchange resins) to extract nuclides using the Dawson-type POM.

The Keggin-type POM (Fig. 3) exhibits excellent Bronsted acidity and interesting redox properties. They can be potentially used for protecting the environment [52]. Reduction-induced $\mathrm{Cs}^{+}$uptake was achieved by the redox-active ionic crystal $\left[\alpha-\mathrm{SiMo}_{12} \mathrm{O}_{40}\right]$ in the presence of ascorbic acid [53]. In a strongly acidic solution, the central sodium ion of the Preyssler anion $\left[\mathrm{NaP}_{5}-\mathrm{W}_{30} \mathrm{O}_{110}\right]^{14-}$ can be replaced by $\mathrm{Eu}^{3+}$ [54]. Therefore, the synergistic effect between POMs and other molecules can assist the efficient removal of nuclides (Fig. 4) [55]. Wang et al. fabricated graphene/POM composites utilizing covalent bonding. Fabrication of the composites exploiting the concept of covalent bonding addressed the problem of POM detachment from the carriers. The report revealed that the surface area of the GO/POM composite was five times larger than that of the POM-only [56]. Lu et al. reported strong interlayer interactions could be produced by POMs (on the GO sheets) when epoxy groups are substituted by ethylenediamine groups (EN, weak reductant). Abundant hopping sites are generated that promote the rapid propagation of protons. It has been reported that the anchoring of POM nanoparticles onto the GO layers helps to generate mobile protons and flexible routes for proton transport. Therefore, the protons can exploit the hopping sites to change their motion path and speed up the process of transportation [57]. The TEM images reveal that the individual clusters of POM appear as black spots on the surface area (when $\mathrm{POM}$ is anchored onto the GO layer). It can be concluded that the oxygen functional group on the graphene sheet and the corner shared metal-oxygen site of POMs in an aqueous solution, interacting with each other via electrostatic interactions [58].

To date, many researchers have attempted to synthesize POM composites that can potentially serve as adsorbents. Herrmann et al. investigated the effects of chemisorption and physisorption of POM-ionic liquid (POM-IL) [59]. Liu et al. investigated these effects in more detail. They successfully fabricated a GO-based nanocomposite by impregnating POM on the GO layer

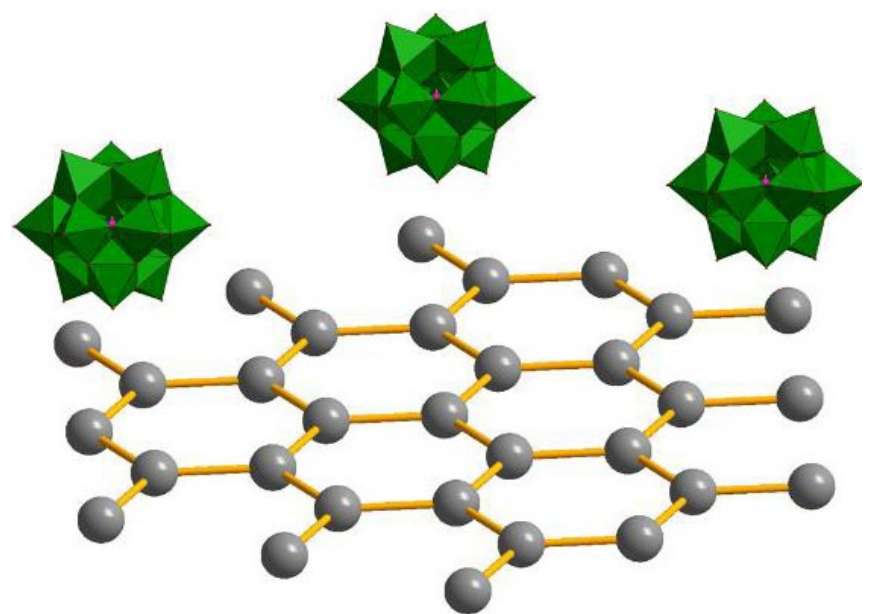

Fig 4. Plausible interactions between Keggin-type POMs and GO

(following the impregnation method), exhibiting an improved loading ability of GO. The fabrication process can potentially lead to the strong electrostatic attraction between the adsorbate and the absorbent [60].

Sures and Nyman carried out their experiments with $\mathrm{A}_{8}\left[\mathrm{Nb}_{6} \mathrm{O}_{19}\right] \cdot \mathrm{nH}_{2} \mathrm{O}(\mathrm{A}=\mathrm{Li}, \mathrm{K}, \mathrm{Rb}, \mathrm{Cs}),\left(\left[\mathrm{Nb}_{2} \mathrm{~W}_{4} \mathrm{O}_{19}\right]^{4-}\right.$, and $\left[\mathrm{Nb}_{4} \mathrm{~W}_{2} \mathrm{O}_{19}\right]^{6-}$. They concluded that Cs exhibits a strong tendency to form complexes with anionic surfaces. The formation of the complexes can also be mediated by a mediation sphere [61]. Hitose and Uchida studied compounds I and II (Wells-Dawson-type POMs, [a$\left.\mathrm{P}_{2} \mathrm{M}_{18} \mathrm{O}_{62}\right]$ ) that exhibit fast adsorption kinetics ( $1 \mathrm{~h}$ to reach equilibrium). They reported that the compounds exhibited an increased cesium uptake capacity [62].

However, it is challenging to deal with radionuclides (in solution and solid surfaces). Radionuclides, such as $\mathrm{Cs}^{+}$that utilize their unusually high coordination number to form complexes, exhibit abnormal behavior (ion-pairing) [61]. The radiation emitted from a radioactive material can potentially lead to the generation of another variable in adsorption materials that can potentially affect their structural stability [63]. Therefore, a better understanding of the mechanism of action of POMs is required to tune and improve the functions of POM-based materials. POM, which has different arrangements, sizes, charges, and structures, can form several types of crystalline structures $[58,64]$. Their unique structures make them 
important to stabilize ligands for redox-active highvalance metallic fragments [65]. The interaction between electrons and alkali metals (during their migration) in POM-based porous crystals can be explained by the redox ion-exchange phenomenon [66]. Thus, the unique characteristics of POMs eventually lead to improved properties of the material formed by the interaction between POM and the support materials.

\section{- CONCLUSION}

It is imperative to gain insight into the mechanism of the formation of POM-integrated GO. The Fukushima accident of 2011 has led to numerous environmental problems. Radionuclides have become one of the significant concerns of many researchers. It is important to design a nanocomposite that can be potentially used to address the issues of radionuclide contamination. A few effective methods that can be followed to achieve these goals are summarized below. (i) Carboxylation, (ii) Covalent bonding, (iii) Impregnation method. Furthermore, the sorption capacity of the adsorbent can be increased by exploiting the concepts of ion trapping, coagulation, electrostatic interaction, and $\mathrm{H}^{+}$-exchange. These methods and radionuclides removal concept offers a bridge between GO and POMs. In addition, through a better understanding of the specific characteristics of its POM-type (Keggin-type, Dawson-type, Preyssler-type) and the structural diversity of GO having different $\mathrm{O} / \mathrm{C}$ ratio, the optimization of the POM and GO properties can potentially generate a new advanced nanocomposite. Specifically, by considering the dynamic nature of the surface area of GO, it can be used as an entry point to design the structure and the function system. For example, researchers have utilized the multiple oxidation method to increase the sorption ability of the adsorbent. Finally, it can help the remediation of contaminated soil by radionuclides.

\section{- ACKNOWLEDGMENTS}

We appreciate the support received from the Phoenix Leader Education Program, Hiroshima University, Japan. The project was funded by research grants (ENEN PLUS PROJECT-EU, Belgium).

\section{- REFERENCES}

[1] Yasunari, T.J., Stohl, A., Hayano, R.S., Burkhart, J.F., Eckhardt, S., and Yasunari, T., 2011, Cesium137 deposition and contamination of Japanese soils due to the Fukushima nuclear accident, Proc. Natl. Acad. Sci. U.S.A., 108 (49), 19530-19534.

[2] Nakanishi, T.M., 2017, Research with radiation and radioisotopes to better understand plant physiology and agricultural consequences of radioactive contamination from the Fukushima Daiichi nuclear accident, J. Radioanal. Nucl. Chem., 311 (2), 947-971.

[3] Tokyo Electric Power Company, 2011, The evaluation status of reactor core damage at Fukushima Daiichi nuclear, power station unit 1 of 3, https://www.tepco.co.jp/en/nu/fukushima-np/ images/handouts_111130_04-e.pdf.

[4] Wang, X., Chen, L., Wang, L., Fan, Q., Pan, D., Li, J., Chi, F., Xie, Y., Yu, S., Xiao, C., Luo, F., Wang, J., Wang, X., Chen, C., Wu, W., Shi, W., Wang, S., and Wang, X., 2019, Synthesis of novel nanomaterials and their application in efficient removal of radionuclides, Sci. China Chem., 62 (8), 933-967.

[5] Nishihara, K., Yamagishi, I., Yasuda, K., Ishimori, K., Tanaka, K., Kuno, T., Inada, S., and Gotoh, Y., 2015, Radionuclide release to stagnant water in the Fukushima-1 nuclear power plant 1, J. Nucl. Sci. Technol., 52 (3), 301-307.

[6] Foreman, M.R.S.J., 2015, An introduction to serious nuclear accident chemistry, Cogent Chem., 1 (1), 1049111.

[7] Uchida, S., and Tagami, K., 2007, Soil-to-plant transfer factors of fallout ${ }^{137} \mathrm{Cs}$ and native ${ }^{133} \mathrm{Cs}$ in various crops collected in Japan, J. Radioanal. Nucl. Chem., 273 (1), 205-210.

[8] Matsuda, N., and Nakashima, S., 2014, Radioactive cesium in water and soil and its adsorption to rice plant (interim report) (in Japanese), Radiat. Saf. Manage., 13 (1), 84-91.

[9] Nguyen, H.T., Tsujimoto, M., and Nakashima, S., 2019, Study on paddy soil in Fukushima using Mössbauer spectroscopy, Hyperfine Interact., 240 (1), 122. 
[10] Koarashi, J., Atarashi-Andoh, M., Matsunaga, T., Sato, T., Nagao, S., and Nagai, H., 2012, Factors affecting vertical distribution of Fukushima accidentderived radiocesium in soil under different land-use conditions, Sci. Total Environ., 431, 392-401.

[11] Tsujimoto, M., Miyashita, S., Nguyen, H.T., and Nakashima, S., 2020, Monthly change in radioactivity concentration of ${ }^{137} \mathrm{Cs},{ }^{134} \mathrm{Cs}$, and ${ }^{40} \mathrm{~K}$ of paddy soil and rice plants in Fukushima Prefecture, Radiat. Saf. Manage., 19, 10-22.

[12] Basuki, T., Miyashita, S., Tsujimoto, M., and Nakashima, S., 2018, Deposition density of ${ }^{134} \mathrm{Cs}$ and ${ }^{137} \mathrm{Cs}$ and particle size distribution of soil and sediment profile in Hibara Lake area, Fukushima: An investigation of ${ }^{134} \mathrm{Cs}$ and ${ }^{137} \mathrm{Cs}$ indirect deposition into lake from surrounding area, J. Radioanal. Nucl. Chem., 316 (3), 1039-1046.

[13] Shizuma, K., Fujikawa, Y., Kurihara, M., and Sakurai, Y., 2018, Identification and temporal decrease of ${ }^{137} \mathrm{Cs}$ and ${ }^{134} \mathrm{Cs}$ in groundwater in Minami-Soma City following the accident at the Fukushima Dai-ichi nuclear power plant, Environ. Pollut., 234, 1-8.

[14] Steinhauser, G., Brandl, A., and Johnson, T.E., 2014, Comparison of the Chernobyl and Fukushima nuclear accidents: A review of the environmental impacts, Sci. Total Environ., 470-471, 800-817.

[15] Ayabe, Y., Hijii, N., and Takenaka, C., 2017, Effects of local-scale decontamination in a secondary forest contaminated after the Fukushima nuclear power plant accident, Environ. Pollut., 228, 344-353.

[16] Koarashi, J., Atarashi-Andoh, M., Nishimura, S., and Muto, K., 2020, Effectiveness of decontamination by litter removal in Japanese forest ecosystems affected by the Fukushima nuclear accident, Sci. Rep., 10 (1), 6614.

[17] IAEA, 2015, The Fukushima Daichi Accident: Technical Volume 5-Post-Accident Recovery, International Atomic Energy Agency, Vienna, https://www-pub.iaea.org/MTCD/Publications/PDF/ AdditionalVolumes/P1710/Pub1710-TV5-Web.pdf.

[18] del Valle, E.M.M., Galán, M.A., and Carbonell, R.G., 2009, Drug delivery technologies: The way forward in the new decade, Ind. Eng. Chem. Res., 48 (5), 2475-2486.

[19] Tabish, M.S., Hanapi, N.S.M., Ibrahim, W.N.W., Saim, N., and Yahaya, N., 2019, Alginate-graphene oxide biocomposite sorbent for rapid and selective extraction of non-steroidal anti-inflammatory drugs using micro-solid phase extraction, Indones. J. Chem., 19 (3), 684-695.

[20] Nor, N.M., Kamil, N.H.N., Mansor, A.I., and Maarof, H.I., 2020, Adsorption analysis of fluoride removal using graphene oxide/eggshell adsorbent, Indones. J. Chem., 20 (3), 579-586.

[21] Sun, Y., Wang, Q., Chen, C., Tan, X., and Wang, X., 2012, Interaction between $\mathrm{Eu}(\mathrm{III})$ and graphene oxide nanosheets investigated by batch and extended X-ray absorption fine structure spectroscopy and by modeling techniques, Environ. Sci. Technol., 46 (11), 6020-6027.

[22] Sun, Y., Shao, D., Chen, C., Yang, S., and Wang, X., 2013, Highly efficient enrichment of radionuclides on graphene oxide-supported polyaniline, Environ. Sci. Technol., 47 (17), 9904-9910.

[23] Ke, Q., and Wang, J., 2016, Graphene-based materials for supercapacitor electrodes - A review, J. Materiomics, 2 (1), 37-54.

[24] Fan, J., Shi, Z., Lian, M., Li, H., and Yin, J., 2013, Mechanically strong graphene oxide/sodium alginate/polyacrylamide nanocomposite hydrogel with improved dye adsorption capacity, J. Mater. Chem. A, 1 (25), 7433-7443.

[25] Debataraja, A., Manurung, R.V., Asri, L.A.T.W., Yuliarto, B., Nugraha, N., and Sunendar, B., 2018, Synthesis and characterization of nanocomposites of tin-oxide graphene doping $\mathrm{Pd}$ using Polyol method, Indones. J. Chem., 18 (2), 344-348.

[26] Eigler, S., Dotzer, C., Hof, F., Bauer, W., and Hirsch, A., 2013, Sulfur species in graphene oxide, Chem. Eur. J., 19 (29), 9490-9496.

[27] Petrov, V.G., Chen, Z., Romanchuk, A.Y., Demina, V.O., Tang, Y., and Kalmykov, S.N., 2019, Sorption of $\mathrm{Eu}$ (III) onto nano-sized H-titanates of different structures, Appl. Sci., 9 (4), 697. 
[28] Tamura, K., Kogure, T., Watanabe, Y., Nagai, C., and Yamada, H., 2014, Uptake of cesium and strontium ions by artificially altered phlogopite, Environ. Sci. Technol., 48 (10), 5808-5815.

[29] Dideikin, A.T., and Vul, A.Y., 2019, Graphene oxide and derivatives: The place in graphene family, Front. Phys., 6, 149.

[30] Szabó, T., Berkesi, O., Forgó, P., Josepovits, K., Sanakis, Y., Petridis, D., and Dékány, I., 2006, Evolution of surface functional groups in a series of progressively oxidized graphite oxides, Chem. Mater., 18 (11), 2740-2749.

[31] Grote, F., Gruber, C., Börrnert, F., Kaiser, U., and Eigler, S., 2017, Thermal disproportionation of oxofunctionalized graphene, Angew. Chem. Int. Ed., 56 (31), 9222-9225.

[32] Hummers, W.S., and Offeman, R.E., 1958, Preparation of graphitic oxide, J. Am. Chem. Soc., 80 (6), 1339.

[33] Ranjan, P., Agrawal, S., Sinha, A., Rao, T.R., Balakrishnan, J., and Thakur, A.D., 2018, A low-cost non-explosive synthesis of graphene oxide for scalable applications, Sci. Rep., 8 (1), 12007.

[34] Cote, L.J., Kim, J., Zhang, Z., Sun, C., and Huang, J., 2010, Tunable assembly of graphene oxide surfactant sheets: Wrinkles, overlaps and impacts on thin film properties, Soft Matter, 6 (24), 6096-6101.

[35] Guerrero-Contreras, J., and Caballero-Briones, F., 2015, Graphene oxide powders with different oxidation degree, prepared by synthesis variations of the Hummers method, Mater. Chem. Phys., 153, 209-220.

[36] Xu, Y., Liu, Z.W., Xu, Y.L., Zhang, Y.Y., and Wu, J.L., 2014, Preparation and characterization of graphene/ZnS nanocomposites via a surfactant-free method, J. Exp. Nanosci., 9 (4), 415-420.

[37] Romanchuk, A.Y., Slesarev, A.S., Kalmykov, S.N., Kosynkin, D.V., and Tour, J.M., 2013, Graphene oxide for effective radionuclide removal, Phys. Chem. Chem. Phys., 15 (7), 2321-2327.

[38] Seliman, A.F., Lasheen, Y.F., Youssief, M.A.E., AboAly, M.M., and Shehata, F.A., 2014, Removal of some radionuclides from contaminated solution using natural clay: Bentonite, J. Radioanal. Nucl. Chem., 300 (3), 969-979.

[39] Mahmoud, M.R., Sharaf El-deen, G.E., and Soliman, M.A., 2014, Surfactant-impregnated activated carbon for enhanced adsorptive removal of $\mathrm{Ce}(\mathrm{IV})$ radionuclides from aqueous solutions, Ann. Nucl. Energy, 72, 134-144.

[40] Kaewmee, P., Manyam, J., Opaprakasit, P., Le, G.T.T., Chanlek, N., and Sreearunothai, P., 2017, Effective removal of cesium by pristine graphene oxide: Performance, characterizations and mechanisms, RSC Adv., 7 (61), 38747-38756.

[41] Semcuk, S., 2018, Application of graphene oxide based nanocomposites and Šaltiškiai clay for radionuclides removal from contaminated solutions, Dissertation, Institute of Physics of the State Research Institute for Physical Science and Technology, Vilnius University, Lithuania.

[42] Yang, H., Sun, L., Zhai, J., Li, H., Zhao, Y., and Yu, H., 2014, In situ controllable synthesis of magnetic Prussian blue/graphene oxide nanocomposites for removal of radioactive cesium in water, J. Mater. Chem. A, 2 (2), 326-332.

[43] Bubeníková, M., Ecorchard, P., Szatmáry, L., Mrózek, O., Salačová, P., and Tolasz, J., 2018, Sorption of $\mathrm{Sr}(\mathrm{II})$ onto nanocomposites of graphene oxide-polymeric matrix, J. Radioanal. Nucl. Chem., 315 (2), 263-272.

[44] Morimoto, N., Kubo, T., and Nishina, Y., 2016, Tailoring the oxygen content of graphite and reduced graphene oxide for specific applications, Sci. Rep., 6 (1), 21715.

[45] Ebajo, V.D., Santos, C.R.L., Alea, G.V., Lin, Y.A., and Chen, C.H., 2019, Regenerable acidity of graphene oxide in promoting multicomponent organic synthesis, Sci. Rep., 9 (1), 15579.

[46] Nováček, M., Jankovský, O., Luxa, J., Sedmidubský, D., Pumera, M., Fila, V., Lhotka, M., Klímová, K., Matějková, S., and Sofer, Z., 2017, Tuning of graphene oxide composition by multiple oxidations for carbon dioxide storage and capture of toxic metals, J. Mater. Chem. A, 5 (6), 2739-2748. 
[47] Xia, T., Qi, Y., Liu, J., Qi, Z., Chen, W., and Wiesner, M.R., 2017, Cation-inhibited transport of graphene oxide nanomaterials in saturated porous media: The Hofmeister effects, Environ. Sci. Technol., 51 (2), 828-837.

[48] Pope, M.T., and Müller, A., 1991, Polyoxometalate chemistry: An old field with new dimensions in several disciplines, Angew. Chem. Int. Ed. Engl., 30 (1), 34-48.

[49] Nyman, M., and Burns, P.C., 2012, A comprehensive comparison of transition- metal and actinyl polyoxometalates, Chem. Soc. Rev., 41 (22), 73547367.

[50] Wihadi, M.N.K., Hayashi, A., Ichihashi, K., Ota, H., Nishihara, S., Inoue, K., Tsunoji, N., Sano, T., and Sadakane, M., 2019, A sandwich complex of bismuth cation and mono-lacunary a-Keggin-type phosphotungstate: Preparation and structural characterization, Eur. J. Inorg. Chem., 2019 (3-4), 357-362.

[51] Hatcher, J.L., 2018, Fundamental chemistry related to separations and coordination of actinium-225, thorium-227, and technetium-99, Dissertation, The Graduate Center, City University of New York, US.

[52] Petit, C., and Bandosz, T.J., 2009, Graphite oxide/polyoxometalate nanocomposites as adsorbents of ammonia, J. Phys. Chem. C, 113 (9), 3800-3809.

[53] Seino, S., Kawahara, R., Ogasawara, Y., Mizuno, N., and Uchida, S., 2016, Reduction-induced highly selective uptake of cesium ions by an ionic crystal based on silicododecamolybdate, Angew. Chem. Int. Ed., 55 (12), 3987-3991.

[54] Kim, K.C., Pope, M.T., Gama, G.J., and Dickman, M.H., 1999, Slow proton exchange in aqueous solution. Consequences of protonation and hydration within the central cavity of Preyssler anion derivatives, $\left[\left|\mathrm{M}\left(\mathrm{H}_{2} \mathrm{O}\right)\right| \supset \mathrm{P}_{5} \mathrm{~W}_{30} \mathrm{O}_{110}\right]^{\mathrm{n}-}$, J. Am. Chem. Soc., 121 (48), 11164-11170.

[55] Li, H., Pang, S., Feng, X., Müllen, K., and Bubeck, C., 2010, Polyoxometalate assisted photoreduction of graphene oxide and its nanocomposite formation, Chem. Commun., 46 (34), 6243-6245.
[56] Wang, R., Dang, L., Liu, Y., and Jiao, W., 2019, Preparation, characterization and photocatalytic activity of Dawson type phosphotungstate/graphene oxide composites, Adv. Powder Technol., 30 (7), 1400-1408.

[57] Liu, Y., Liu, S., Lai, X., Miao, J., He, D., Li, N., Luo, F., Shi, Z., and Liu, S., 2015, Polyoxometalatemodified sponge-like graphene oxide monolith with high proton-conducting performance, $A d v$. Funct. Mater., 25 (28), 4480-4485.

[58] Kim, Y., and Shanmugam, S., 2013, Polyoxometalate-reduced graphene oxide hybrid catalyst: Synthesis, structure, and electrochemical properties, ACS Appl. Mater. Interfaces, 5 (22), 12197-12204.

[59] Herrmann, S., De Matteis, L., de la Fuente, J.M., Mitchell, S.G., and Streb, C., 2017, Removal of multiple contaminants from water by polyoxometalate supported ionic liquid phases (POM-SILPs), Angew. Chem. Int. Ed., 56 (6), 16671670.

[60] Liu, Y., Luo, F., Liu, S., Liu, S., Lai, X., Li, X., Lu, Y., Li, Y., Hu, C., Shi, Z., and Zheng, Z., 2017, Aminated graphene oxide impregnated with photocatalytic polyoxometalate for efficient adsorption of dye pollutants and its facile and complete photoregeneration, Small, 13 (4), 1603174.

[61] Sures, D.J., and Nyman, M., 2017, "Anomalous cesium ion behavior in aqueous polyoxometalate solutions" in Cesium: Properties, Production and Application, Eds. Hall, B., Nova Science Publisher, Inc., New York, 119-148.

[62] Hitose, S., and Uchida, S., 2018, Rapid uptake/release of $\mathrm{Cs}^{+}$in isostructural redox-active porous ionic crystals with large-molecular-size and easily reducible Dawson-type polyoxometalates as building blocks, Inorg. Chem., 57 (9), 4833-4836.

[63] Belloni, F., Kütahyali, C., Rondinella, V.V., Carbol, P., Wiss, T., and Mangione, A., 2009, Can carbon nanotubes play a role in the field of nuclear waste management?, Environ. Sci. Technol., 43 (5), 12501255. 
[64] Ammam, M., 2013, Polyoxometalates: Formation, structures, principal properties, main deposition, methods and application in sensing, J. Mater. Chem. A, 1 (21), 6291-6312.

[65] Miras, H.N., Yan, J., Long, D.L., and Cronin, L., 2012, Engineering polyoxometalates with emergent properties, Chem. Soc. Rev., 41 (22), 7403-7430.

[66] Kawahara, R., Uchida, S., and Mizuno, N., 2015, Redox-induced reversible uptake-release of cations in porous ionic crystals based on polyoxometalate: Cooperative migration of electrons with alkali metal ions, Chem. Mater., 27 (6), 2092-2099. 